\title{
Management of Trigeminal Neuralgia Caused by an Intraneural Spiral Trigeminocerebellar Artery: A Case Report
}

\author{
Naoki Wakuta ${ }^{1}$ Hiroshi Abe ${ }^{1}$ Masani Nonaka ${ }^{1}$ Toshio Higashi ${ }^{1}$ Tetsuya Ueba ${ }^{2}$ Tooru Inoue ${ }^{1}$ \\ ${ }^{1}$ Department of Neurosurgery, Faculty of Medicine, Fukuoka \\ University, Fukuoka, Japan \\ 2 Department of Neurosurgery, Faculty of Medicine, Kochi University, \\ Kochi, Japan

\begin{abstract}
Address for correspondence Naoki Wakuta, MD, Department of Neurosurgery, Faculty of Medicine, Fukuoka University, 7-45-1 Nanakuma, Jounan-ku, Fukuoka 814-0180, Japan

(e-mail: n.wakuta@hotmail.com).
\end{abstract}

J Neurol Surg Rep 2015;76:e59-e61.

\author{
Abstract \\ Keywords \\ - trigeminal neuralgia \\ - trigeminocerebellar \\ artery \\ - intraneural vessel \\ - reshaping
}

The trigeminocerebellar artery (TCA) is a branch of the basilar artery that may have an intraneural course and may cause trigeminal neuralgia. We report a case of trigeminal neuralgia with right vertebral artery aneurysm caused by an intraneural TCA that compressed the trigeminal nerve in multiple places. We performed proximal trapping for the fusiform aneurysm with extra-intracranial bypass to preserve flow of the posterior inferior cerebellar artery, followed by microvascular decompression that successfully changed the course of the TCA. This procedure provided relief from the neuralgia without direct bisection of the trigeminal nerve that may cause severe nerve injury. Reshaping of the course of the artery can achieve good pain relief.

\section{Introduction}

Trigeminal neuralgia is characterized by paroxysmal facial pain. ${ }^{1}$ Dandy $^{2}$ reported that this condition is caused by vascular compression of the trigeminal nerve in the posterior fossa, and Jannetta ${ }^{3}$ described treatment by microvascular decompression. The vessel causing compression of the nerve is often the superior cerebellar artery, ${ }^{4}$ but in some cases compression is caused by a trigeminocerebellar artery (TCA) with an unusual course. ${ }^{5-8}$ Vessel transposition techniques have been developed to relieve external compression of the nerve. ${ }^{9}$ However, management of trigeminal neuralgia caused by an intraneural artery has rarely been reported, ${ }^{10,11}$ and the optimal treatment strategy for this condition remains unclear. We describe a unique case of trigeminal neuralgia caused by an intraneural spiral TCA with compression of the trigeminal nerve in multiple places.

\section{Case Report}

Our patient was a 70-year-old woman with a 5 -year history of typical trigeminal neuralgia, with paroxysmal lancinating facial pain in the area supplied by the second division of the right trigeminal nerve. She described the pain as sharp and like an electrical shock, lasting from a few seconds to a minute. The trigger zone was over the right infraorbital foramen. Her symptoms were initially well controlled by carbamazepine, but the frequency and severity of episodes gradually increased. Thin-cut magnetic resonance imaging (MRI) showed compression of the right trigeminal nerve in the cerebellopontine cistern by the right TCA ( - Fig. $\mathbf{1}$ ). MRI and cerebral angiography showed a fusiform aneurysm of the right vertebral artery that had no relationship with the trigeminal nerve (-Fig. 2).

We performed proximal trapping of the aneurysm following extra-intracranial bypass, with anastomosis of the occipital artery to the posterior inferior cerebellar artery. Craniotomy was performed via the lateral suboccipital approach. After completion of surgery for the aneurysm, we approached the root entry zone of the trigeminal nerve along the transverse-sigmoid junction with gentle cerebellar retraction. Initially, it appeared that the TCA compressed only the superior aspect of the trigeminal nerve. However, arachnoidal dissection around the artery-nerve complex revealed that the received

June 15, 2014

accepted after revision

October 22, 2014

published online

May 13, 2015
DOI http://dx.doi.org/

10.1055/s-0034-1543975. ISSN 2193-6366. (c) 2015 Georg Thieme Verlag KG
Stuttgart · New York

License terms

((1) $\Theta \circledast$ 

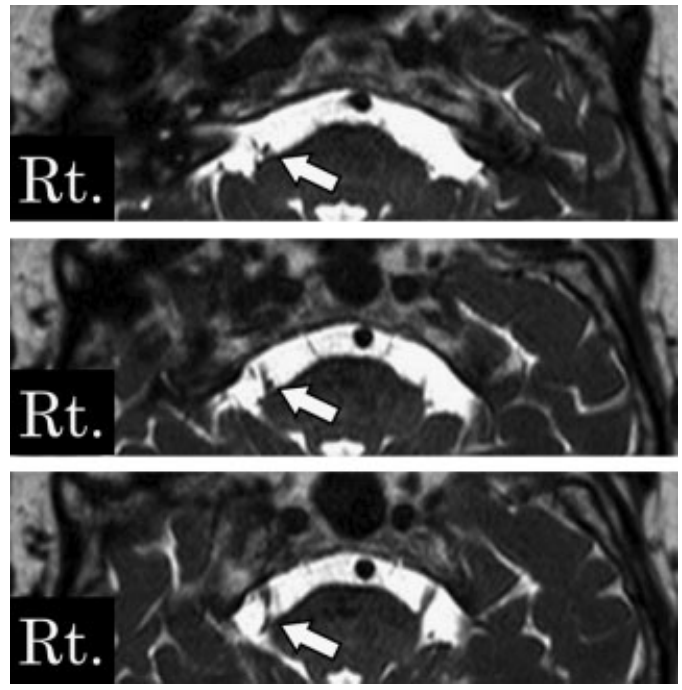

Fig. 1 Serial thin-slice T2-weighted magnetic resonance images (axial view) showing the trigeminocerebellar artery as a flow void adjacent to the right trigeminal nerve in the prepontine cistern (arrows).

TCA twisted around the trigeminal nerve like a corkscrew and passed between the sensory (portio major) and motor (portio minor) roots of the nerve ( $\mathbf{- F i g} . \mathbf{3 A - C}) \cdot{ }^{12}$ Multiple indentations of the trigeminal nerve were observed $(-$ Fig. $3 \mathbf{C})$. The TCA was gently straightened and mobilized, and its course was reshaped to avoid compression of the trigeminal nerve ( - Fig. 3D). The TCA was then attached to the dura with Teflon felt and fibrin glue. A piece of Teflon felt was also interposed between the intraneural portion of the TCA and the trigeminal nerve. Postoperatively, the patient's trigeminal neuralgia symptoms resolved immediately.

\section{Discussion}

During surgery for trigeminal neuralgia, the vessel causing compression can usually be identified. The superior cerebellar artery is responsible for $75 \%$ of cases. ${ }^{4}$ In some patients an unusual course of the TCA, a branch of the basilar artery, causes compression of the trigeminal nerve. ${ }^{5-8}$ In cadaveric
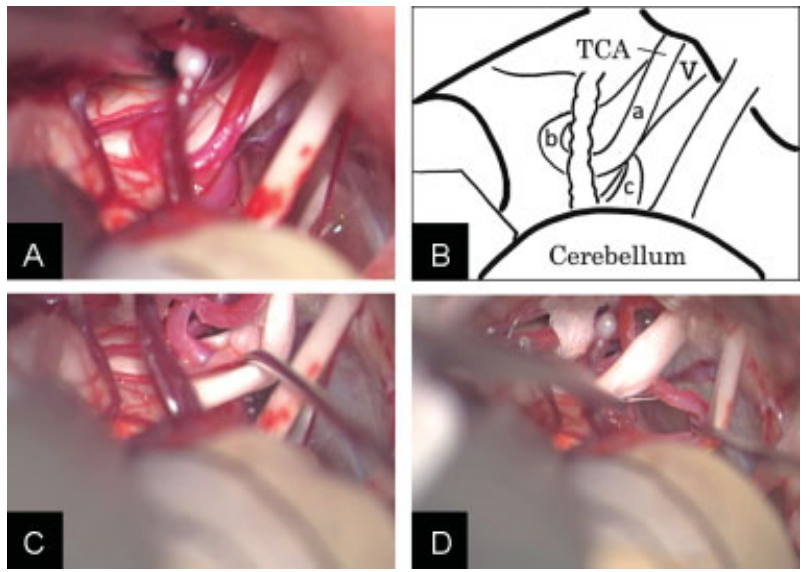

Fig. 3 (A, C, D) Intraoperative photographs and (B) corresponding illustration. (A) The trigeminocerebellar artery (TCA) had a spiral course around the trigeminal nerve $(V)$. (B) Illustration of the view in (A) showing the course of the TCA, with successive parts (proximal to distal) marked in alphabetical order. (C) Indentation of the trigeminal nerve was revealed after dissection to allow observation of the intraneural portion of the TCA. (D) The surgical procedure changed the course of the TCA to relieve the compression of the trigeminal nerve.

studies, the reported frequency of a close relationship between the TCA and the trigeminal nerve is 2.3 to $13.3 \%$, 5,13 The TCA sometimes twists around or encircles the trigeminal nerve $^{7}$ and may thereby cause trigeminal neuralgia. The TCA has been reported to penetrate the root entry zone of the trigeminal nerve in some patients with trigeminal neuralgia, but the prevalence of this configuration is unknown. ${ }^{5,7}$ The reasons for the development of such intraneural vessels are not clear. During neural differentiation, the ophthalmic and maxillomandibular nerves fuse before development of the three divisions of the trigeminal nerve. It may be that the vessels get trapped between the nerves during the fusion process, resulting in an intraneural course. ${ }^{14}$

Intraneural vessels are very difficult to treat because aggressive surgery can lead to severe complications. ${ }^{10,11,14}$ Helbig et al ${ }^{14}$ reported that intraneural veins were exposed during $2 \%$ of microvascular decompression procedures. Only two previous reports have described patients with trigeminal
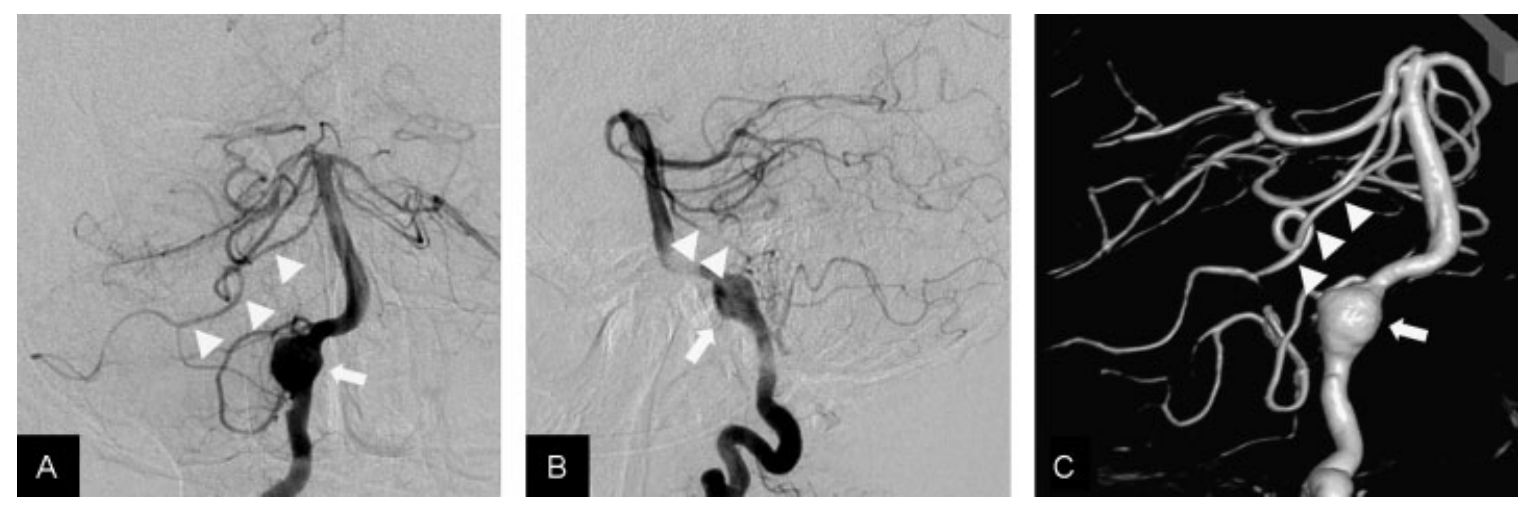

Fig. 2 Right vertebral angiography showing the right trigeminocerebellar artery (TCA) (arrowheads) and the fusiform aneurysm (arrows) of the right vertebral artery. (A) Anteroposterior view. (B) Lateral view. Three-dimensional digital subtraction angiography showing the spiral course of the TCA. (C) Right anterior oblique view. 
neuralgia associated with an intraneural artery. ${ }^{10,11}$ This is the first report of a patient who underwent surgery for trigeminal neuralgia caused by a spiral intraneural TCA with compression of the nerve in multiple places.

Two previous reports including 11 patients described trigeminal neuralgia caused by intraneural veins. ${ }^{11,14}$ The offending vein was sacrificed in nine patients, and the other two patients were treated by interposition of Teflon implants between the vein and the nerve. Facial pain was relieved in all patients, but three developed facial numbness and one developed dense corneal hypoesthesia. Patrick ${ }^{10}$ reported a patient with trigeminal neuralgia caused by an intraneural anterior inferior cerebellar artery who was treated by separation of the trigeminal nerve from the artery. Postoperatively, the patient was pain free for 6 months but required subsequent percutaneous thermocoagulation of the trigeminal nerve to control recurrent pain. Zheng et al $^{11}$ reported three patients who were treated by wrapping the intraneural artery with a piece of Teflon sponge. These patients all experienced resolution of their pain, but one developed facial numbness.

If the vessel causing trigeminal neuralgia is a major artery, as in our patient, it is very challenging to perform dissection around the artery. ${ }^{11,14}$ If the spiral course of the vessel causes both internal and external compression of the nerve, wrapping of the intraneural portion with Teflon felt may be insufficient and may even lead to exacerbation by increasing the compression. In our patient, we straightened the TCA and changed its course, thereby resolving the complex combination of external and intraneural compression. This surgical technique did not require an invasive procedure such as enlargement of the passage by direct bisection of the trigeminal nerve, and the patient's facial pain was relieved with no complications.

To confirm the safety of this procedure, further data should be collected to clarify the pathologic role of intraneural arteries in trigeminal neuralgia and the perioperative complications and postoperative recurrence rate.

\section{Conclusion}

We present the first reported case of trigeminal neuralgia caused by multiple areas of compression by an intraneural spiral TCA. This condition seems difficult to treat using standard microvascular decompression techniques, and aggressive surgery carries a risk of nerve injury. Pain relief was safely achieved by reshaping the course of the artery.

\section{References}

1 Greenberg MS. Trigeminal neuralgia. In: Handbook of Neurosurgery. New York, NY: Thieme; 2006:378-386

2 Dandy WE. The treatment of trigeminal neuralgia by the cerebellar route. Ann Surg 1932;96(4):787-795

3 Jannetta PJ. Arterial compression of the trigeminal nerve at the pons in patients with trigeminal neuralgia. J Neurosurg 1967;26 (1, Suppl):159-162

4 Jannetta PJ. A comprehensive reference guide to the diagnosis and management of neurological problems. Cranial rhizopathies. In: Youmans JR, ed. Neurological Surgery. Philadelphia, PA: WB Saunders; 1990:4169-4182

5 Marinković S, Gibo H, Nikodijević I. Trigeminocerebellar arteryanatomy and possible clinical significance. Neurol Med Chir (Tokyo) 1996;36(4):215-219

6 Stephens RB, Stilwell DL. Arteries and Veins of the Human Brain. Springfield, IL: CC Thomas; 1969:71-123

7 Tuccar E, Sen T, Esmer AF. Anatomy and clinical significance of the trigeminocerebellar artery. J Clin Neurosci 2009;16(5): 679-682

8 Willinsky R, Lasjaunias P, Berenstein A. Intracavernous branches of the internal carotid artery (ICA). Comprehensive review of their variations. Surg Radiol Anat 1987;9(3):201-215

9 Barker FG II, Jannetta PJ, Bissonette DJ, Larkins MV, Jho HD. The long-term outcome of microvascular decompression for trigeminal neuralgia. N Engl J Med 1996;334(17):1077-1083

10 Patrick BS. Unusual nerve-artery relationship in microvascular exploration for tic douloureux. Neurosurgery 2005;56(3):E629

11 Zheng X, Feng B, Hong W, et al. Management of intraneural vessels during microvascular decompression surgery for trigeminal neuralgia. World Neurosurg 2012;77(5-6):771-774

12 Gudmundsson K, Rhoton AL Jr, Rushton JG. Detailed anatomy of the intracranial portion of the trigeminal nerve. J Neurosurg 1971; 35(5):592-600

13 Marinković SV, Gibo H. The blood supply of the trigeminal nerve root, with special reference to the trigeminocerebellar artery. Neurosurgery 1995;37(2):309-317

14 Helbig GM, Callahan JD, Cohen-Gadol AA. Variant intraneural veintrigeminal nerve relationships: an observation during microvascular decompression surgery for trigeminal neuralgia. Neurosurgery 2009;65(5):958-961; discussion 961 